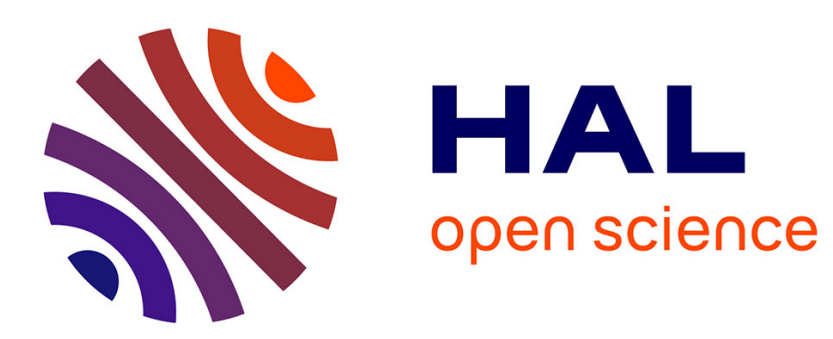

\title{
Exact properties of spin glasses. II. Nishimori's line: new results and physical implications
}

\author{
A. Georges, D. Hansel, P. Le Doussal, J.-P. Bouchaud
}

\section{To cite this version:}

A. Georges, D. Hansel, P. Le Doussal, J.-P. Bouchaud. Exact properties of spin glasses. II. Nishimori's line: new results and physical implications. Journal de Physique, 1985, 46 (11), pp.1827-1836. 10.1051/jphys:0198500460110182700 . jpa-00210133

\section{HAL Id: jpa-00210133 https://hal.science/jpa-00210133}

Submitted on 1 Jan 1985

HAL is a multi-disciplinary open access archive for the deposit and dissemination of scientific research documents, whether they are published or not. The documents may come from teaching and research institutions in France or abroad, or from public or private research centers.
L'archive ouverte pluridisciplinaire HAL, est destinée au dépôt et à la diffusion de documents scientifiques de niveau recherche, publiés ou non, émanant des établissements d'enseignement et de recherche français ou étrangers, des laboratoires publics ou privés. 
Classification

Physics Abstracts

$75.40-05.50$

\title{
Exact properties of spin glasses. II. Nishimori's line : new results and physical implications
}

\author{
A. Georges \\ Laboratoire de Physique Théorique de l'Ecole Normale Supérieure (*), \\ 24, rue Lhomond, 75231 Paris Cedex, France \\ D. Hansel
}

Centre de Physique Théorique de l'Ecole Polytechnique (**), 91128 Palaiseau Cedex, France

\section{P. Le Doussal}

Laboratoire de Physique Théorique de l'Ecole Normale Supérieure (*), 24, rue Lhomond, 75231 Paris Cedex, France

and J.-P. Bouchaud

Laboratoire de Spectroscopie Hertzienne de l'Ecole Normale Supérieure (***), 24, rue Lhomond, 75231 Paris Cedex, France

(Reçu le 14 mai 1985, accepté le 10 juillet 1985)

\begin{abstract}
Résumé. - Nous réexaminons le résultat exact de Nishimori pour les verres de spin. L'existence d'un sous-espace du diagramme des phases, où des propriétés exactes peuvent être obtenues, est étendue à une classe de modèles plus générale. Ceci clarifie le rôle de la symétrie de jauge. Nous obtenons une nouvelle borne supérieure pour l'énergie libre sur ce sous-espace. Nous confrontons ces résultats à des simulations numériques récentes pour les modèles S.K. et Ising en dimension 2 et 3. Dans le cas bidimensionnel cela suggère l'existence possible d'un point tricritique à $p_{\mathrm{c}} \simeq 0,9, \frac{k T_{\mathrm{c}}}{\mathrm{J}} \simeq 0,9$.

Abstract. - An exact result for spin glasses, due to Nishimori, is reconsidered. The existence, in the phase diagram, of a subspace where some exact properties can be derived, is generalized to a larger class of models. This clarifies the role of the gauge symmetry. A new upper bound for the quenched free energy on this subspace is obtained. Recent numerical simulations are confronted with these results for S.K., 3d and $2 \mathrm{~d}$ Ising spin glasses. This suggests the possible existence of a tricritical point for the $2 \mathrm{~d}$ Ising spin glass at $p_{\mathrm{c}} \simeq 0.9, \frac{k T_{\mathrm{c}}}{\mathrm{J}} \simeq 0.9$.
\end{abstract}

\section{Introduction.}

In statistical mechanics of pure systems, a large class of models can be solved exactly. Moreover, exact

(*) Laboratoire Propre du Centre National de la Recherche Scientifique, associé à l'Ecole Normale Supérieure et à l'Université de Paris Sud.

(**) Groupe de Recherche du Centre National de la Recherche Scientifique $n^{\circ} 48$.

$\left({ }^{* * *}\right)$ Laboratoire Associé au Centre National de la Recherche Scientifique. results can be derived, even for some non-integrable models, providing powerful information on their physical properties.

Unfortunately, the situation is different for the spin glass problem, where very few exact properties are available to guide theoretical approaches. A remarkable exception is the discovery by Nishimori $[1,2]$, for spin glasses of Ising type, of a subspace in the phase diagram where an exact expression is available for the quenched internal energy. If one defines $p\left(J_{i j}\right)$ as the probability distribution over the random bonds, 
the equation of the subspace reads

$$
\frac{p\left(-J_{i j}\right)}{p\left(J_{i j}\right)}=\mathrm{e}^{-2 \beta J_{i j}}
$$

and the energy takes the surprisingly simple form :

$$
U=-N_{\mathbf{B}}\langle J\rangle
$$

where $N_{\mathbf{B}}$ is the number of bonds.

Other exact results can be derived on the line, such as an upper bound on the specific heat.

In a companion paper [3] we introduced, for the two-dimensional case, a supersymmetric effective theory which undergoes a dimensional reduction on Nishimori's line. There, we also noticed the features common to Nishimori's result and to the « disorder solutions " [21] encountered for pure systems.

In this paper we first extend Nishimori's result to a general gauge invariant spin glass. This emphasizes the role of the gauge symmetry in a more explicit way than in the special case of $\mathbb{Z}_{2}$. We also derive a new upper bound for the free energy on the subspace, which turns out to be very stringent. We then turn to specific models of physical interest. We compare the strong constraints due to these exact results with recent numerical and analytical works. This provides valuable information on the structure of phase diagrams. We take a particular interest in the S.K. model, $3 \mathrm{~d}$ and $2 \mathrm{~d}$ Ising spin glasses. This paper is organized as follows : section 2 is rather formal : we generalize there Nishimori's result to an arbitrary gauge group and give the new bound on $F$. In section 3, we apply our methods to the special case of $\mathbb{Z}_{2}$ (Ising model) and $\mathbb{Z}_{q}$ (Potts model). Section 4 is devoted to the physical discussion of the structure of phase diagrams.

\section{Exact results for gauge invariant spin glasses.}

2.1 A GENERAL MODEL. - In this section, we consider a spin glass model with an arbitrary group $\mathrm{G}$ of local invariance, supposed, for the moment, to be Abelian. This includes the Ising model $\left(G=\mathbb{Z}_{2}\right)$ as well as generalized Potts models $\left(G=\mathbb{Z}_{q}\right)$. We associate an element $g_{i}$ of the group $\mathrm{G}$ with each of the $N$ sites of a lattice. For each interacting pair, we introduce a real valued function $J_{i j}$ on the group G. The Hamiltonian of the model is :

$$
H=-\sum_{(i j)} J_{i j}\left(g_{i} g_{j}^{-1}\right)
$$

where the sum goes over all interacting pairs. The interaction between two sites depends only on the "distance" between the two group elements they carry. As a consequence, $H$ is invariant under the following gauge transformation :

$$
g_{i} \rightarrow \xi_{i} g_{i} \quad J_{i j} \rightarrow \xi_{i} \xi_{j}^{-1} J_{i j}
$$

where $\left\{\xi_{i}\right\}$ is a set of $N$ arbitrary group elements, and the function ${ }^{\xi} J$ is defined by

$$
\xi J(g)=J\left(\xi^{-1} g\right) .
$$

The corresponding random bond model is specified by a probability law $P\left[J_{i j}\right]$ which is a functional of the interactions. Hence, the quenched mean value of a physical quantity $O$ is

$$
\langle O\rangle=\int \prod_{(i j)} P\left[J_{i j}\right] D J_{i j} \frac{\sum_{\{g k\}} O \mathrm{e}^{-\beta H}}{\sum_{\{g k\}} \mathrm{e}^{-\beta H}}
$$

where $D J_{i j}$ is the functional measure over the bonds.

As an illustration, let us show how one recovers the usual Ising spin glass in the case $G=\mathbb{Z}_{2}$. The function $J_{i j}$ is then defined by its two independent values $J_{i j}(+), J_{i j}(-)$. However, the trace $J_{i j}(+)+J_{i j}(-)$ is irrelevant, since (2.1) can be written here as :

$$
\begin{aligned}
H=-\sum_{(i j)} \frac{1}{2}\left(J_{i j}(+)\right. & \left.+J_{i j}(-)\right)- \\
& -\sum_{(i j)} \frac{1}{2}\left(J_{i j}(+)-J_{i j}(-)\right) S_{i} S_{j} .
\end{aligned}
$$

Thus one may consider only probability distributions of the form :

$$
P\left[J_{i j}\right]=p\left(J_{i j}(+)\right) \delta\left(J_{i j}(+)+J_{i j}(-)\right) .
$$

The gauge symmetry $J_{i j}(+) \leftrightarrow J_{i j}(-)$ simply amounts to the change of sign of the usual Ising coupling constant. However, this usual form of the $\mathbb{Z}_{2}$ symmetry is not well suited to generalizations, and conceals the role of the gauge invariance.

In the case $G=\mathbb{Z}_{q}$ our model is a generalized disordered Potts model. If one represents the $q$ states on the unit circle, the Hamiltonian (2.1) reads :

$$
H=-\sum_{(i j)} J_{i j}\left(\theta_{i}-\theta_{j}\right) .
$$

The function $J_{i j}(x)$ is specified by the values it takes on its $q$ possible arguments and depends only on the angle between two states. For this model the gauge invariance is :

$$
\begin{aligned}
\theta_{i} & \rightarrow \theta_{i}+\varphi_{i} \\
J_{i j}(\theta) & \rightarrow J_{i j}\left(\theta+\varphi_{j}-\varphi_{i}\right) .
\end{aligned}
$$

2.2 Generalization OF Nishimori's Results. We first notice that the condition (1.1), in the case of $\mathbb{Z}_{2}$, can be reformulated in the language of subsection 2.1 , as follows : when the probability distribution $P\left[J_{i j}\right]$ can be written as

$$
P\left[J_{i j}\right]=\phi\left(J_{i j}(+), J_{i j}(-)\right) \mathrm{e}^{a J_{i j}(+)}
$$

where $\phi$ is symmetric in its arguments and $a$ is a parameter, then Nishimori's subspace is defined by 
$a=\beta$. The original condition (1.1) for $p$ is recovered under the constraint (2.6).

In the same way we consider, in the following, probability distributions which can be written as :

$$
P\left[J_{i j}\right]=\phi\left[J_{i j}\right] \mathrm{e}^{a J_{i j}(e)}
$$

where $e$ is the neutral element of $\mathrm{G}$, and $\phi\left[J_{i j}\right]$ is a functional such that $\phi\left[{ }^{g} J_{i j}\right]=\phi\left[J_{i j}\right]$ for all group elements $g$.

We can now state the generalization of Nishimori's results. On the subspace of the phase diagram defined by :

$$
a=\beta
$$

one can show the following properties :

i) the quenched internal energy can be calculated exactly and reads :

$U=-N_{\mathbf{B}} \int J(e) P[J] D J=-N_{\mathrm{B}}\langle J(e)\rangle$

ii) the specific heat is bounded :

$$
\langle C\rangle \leqslant N_{\mathrm{B}} \beta^{2}\left(\left\langle J(e)^{2}\right\rangle-\langle J(e)\rangle^{2}\right)
$$

iii) if one defines a « magnetic » order parameter $m$ and a «spin glass" order parameter $Q$ (see Appen$\operatorname{dix} A)$, then :

$$
m=Q
$$

iv) let $p_{1}, \ldots, p_{n}$ be the parameters characterizing the probability distribution (2.10). We consider a point of the subspace (2.11) defined by a given set of values of $p_{1}, \ldots, p_{n}$ and by the corresponding temperature $\beta^{*}=a\left(p_{1}, \ldots, p_{n}\right)$. If this point lies in the paramagnetic phase $(m=0, Q=0)$, then the magnetic order parameter $m\left(\beta ; p_{1}, \ldots, p_{n}\right)$ vanishes for all temperatures.

2.3 A NEW RIGOROUS BOUND ON THE FREE ENERGY. It is remarkable that the quenched mean value of $Z^{-1}$ can be calculated exactly on the subspace (2.11). As shown in Appendix A, it reads :

$$
\left\langle Z^{-1}\right\rangle=\frac{1}{V_{\mathrm{G}}^{N}}\left\langle\mathrm{e}^{-\beta J(e)}\right\rangle^{N_{\mathbf{B}}}
$$

where $V_{\mathrm{G}}$ is the " volume " of the gauge group. Using the convexity of the logarithm :

$$
-\langle\ln Z\rangle \leqslant \ln \left\langle Z^{-1}\right\rangle
$$

we obtain a rigorous upper bound $F_{\mathrm{S}}$ on the quenched free energy $F$ on the subspace :

$$
F_{\mathrm{S}}=-N T \ln V_{\mathrm{G}}+N_{\mathrm{B}} T \ln \left\langle\mathrm{e}^{-\beta J(e)}\right\rangle .
$$

However, the physical requirement of a positive entropy provides another upper bound : the internal energy $U$ given by (2.12). We shall see in section 4 that, for models of physical interest, these bounds are separately relevant in two different regions of the phase diagram. Moreover, when an explicit expression for the annealed free energy is available, it provides a lower bound for $F$. The resulting constraints turn out to be very stringent. In the particular case of the S.K. model they even provide an exact determination of $F$ on Nishimori's line in the paramagnetic phase. This will be discussed in section 4 .

\section{Discussion.}

In this section, we discuss the meaning of these results, and show how the general formalism of section 2 applies to the case of $\mathbb{Z}_{q}$ (generalized Potts model).

3.1 MeAning OF THE Results. - The dimension of the phase diagram of the model $(2.1)$ is simply the number $p$ of parameters characterizing the probability distribution $P$. (Indeed $p$ adimensional quantities can be formed with the temperature and these parameters). When $P$ satisfies (2.10), the condition (2.11) defines a subspace of dimension $p-1$. Specific examples will be given in section 3.2.

On this subspace, the quenched internal energy takes the simple form (2.12), which is the energy of the system when all group elements have the same value at each site. However, this "frozen system " picture is not relevant to all other physical quantities, such as the quenched free energy and magnetization. The expression of $U$ on the subspace is regular for all temperatures, which raises the question of the intersection with the critical lines. While no general answer has been given yet, this point will be considered in section 4 for specific examples.

Let us stress that these results cannot be interpreted as an identification on the subspace of the quenched internal energy with the annealed one. This is easily seen on the example of the one-dimensional Ising spin glass with a finite number of sites and periodic boundary conditions, previously considered in paper $I$ [3].

The symmetries of the phase diagram clearly appear in our formulation. Let us consider a group element $g_{0}$ such that a configuration $\left\{g_{i}^{*}\right\}$ can be implemented on the lattice, in such a way that :

$$
g_{i}^{*} g_{j}^{*-1}=g_{0}
$$

for each ordered pair ( $i j)$ of interacting sites. Then $J_{i j} \rightarrow{ }^{g_{0}} J_{i j}$ leaves $U=\langle H\rangle$ invariant. The corresponding transformation on the parameters characterizing the distribution $P$, defines a symmetry of the phase diagram. On the subspace obtained from (2.11) by this symmetry the internal energy takes the same simple form (2.12). This expression can also be viewed as the energy of the system frozen in the configuration $\left\{g_{i}^{*}\right\}$ but with the transformed probability distribution ${ }^{g_{0}} P$. Let us note that, for $g_{0} \neq e$, the condition (3.1) depends on the particular topology of the lattice : it generalizes the concept of 
an unfrustrated configuration introduced in the case $\mathbf{G}=\mathbb{Z}_{2}$.

Let us also mention that the simplification of $U$ can be understood for an arbitrary group as in paper I for $G=\mathbb{Z}_{2}$ [3]. The function $\mathcal{U}_{N}^{*}(J)$ introduced there can be generalized here as the density associated with

$H+\sum_{(i, j)} J_{i j}(e)$ as, $\int D J P[J] \mathcal{U}_{N}^{*}(J)=\left\langle H+\sum_{(i, j)} J_{i j}(e)\right\rangle$.

On the subspace $a=\beta$ one can show that :

$$
\sum_{g \in G} e^{\beta J(g)} \mathcal{U}_{N}^{*}\left[{ }^{g} J\right]=0 .
$$

The simplification of the internal energy then appears as a cancellation on the average between the contributions to $\left\langle H+\sum_{(i j)} J_{i j}(e)\right\rangle$ of the system with one bond frozen to a given value, and frozen to its gauge transformed values.

Finally, we emphasize that the results of section 2 can be extended with minimal changes to a nonAbelian group.

\subsection{ApPLICATION to $\mathbb{Z}_{2}$ AND $\mathbb{Z}_{q}$. -}

$+\mathrm{G}=\mathbb{Z}_{2}$ (Ising model).

We have seen in subsection 2.1 that, in this case, one can consider only probability distributions of the form (2.6). The condition (2.11) is then simply the original definition (1.1) of Nishimori's subspace. For the sake of completeness, we recall how it applies to some distributions of physical interest :

(Gaussian distribution)

$$
p\left(J_{i j}\right)=\frac{1}{\sqrt{2 \pi} J} \exp \left[-\left(J_{i j}-J_{0}\right)^{2} / 2 J^{2}\right]
$$

(Binary distribution)

$$
p\left(J_{i j}\right)=p \delta\left(J_{i j}-J\right)+(1-p) \delta\left(J_{i j}+J\right) .
$$

Nishimori's subspace is a line of the two-dimensional phase diagram, which reads :

$$
\beta J^{2}=J_{0}, \quad U=-N_{\mathrm{B}} J_{0}
$$

(BD) $2 p-1=\tanh \beta J, U=-N_{\mathrm{B}}(2 p-1) J$.

For the dilute binary model :

$p\left(J_{i j}\right)=q p \delta\left(J_{i j}-J\right)+q(1-p) \delta\left(J_{i j}+J\right)+(1-q) \delta\left(J_{i j}\right)$

the subspace becomes a surface in the three-dimensional phase diagram, defined by the same equation as in the binary case.
$+\mathrm{G}=\mathbb{Z}_{q}$ (generalized Potts model).

As in (2.7), we represent the $q$ states by the angles $\theta_{k}=2 \pi k / q(=1, \ldots, q)$. For each bond, the function $J$ is completely specified by its $q$ values $J\left(\theta_{k}\right)$, denoted simply by $J_{k}$ in the following. The general form (2.10) of the distribution $P$ to which our results apply, reads here :

$$
P\left(J_{1}, \ldots, J_{q}\right)=\phi\left(J_{1}, \ldots, J_{q}\right) \mathrm{e}^{a J_{q}}
$$

where $\phi$ is invariant under the cyclic permutations of its arguments. However, for most physical situations, it is sufficient to consider $\phi$ functions invariant under all permutations.

We first look for splitted probability distributions, corresponding to independent $J_{k}$ 's. (3.8) then constrains $P$ to be of the form :

$$
P\left(J_{1}, \ldots, J_{q}\right)=C p\left(J_{q}\right) \mathrm{e}^{a J_{q}} \prod_{k=1}^{q-1} p\left(J_{k}\right)
$$

where $C$ is a normalization constant. As a first example, let us take a binary distribution (3.4) for $p\left(J_{k}\right)$, of mean value $J$ and probability $p$. This corresponds to a binary distribution for $J_{q}$ characterized by the same mean value $J$ and a probability $p^{\prime}$, so that $a$ is related to $p$ and $p^{\prime}$ by :

$$
p^{\prime}=\frac{p \mathrm{e}^{a J}}{p \mathrm{e}^{a J}+(1-p) \mathrm{e}^{-a J}} .
$$

The condition $a=\beta$ reads :

$$
\frac{1-p^{\prime}}{p^{\prime}}=\frac{1-p}{p} \mathrm{e}^{-2 \beta J}
$$

This defines a surface in the diagram $\left(p, p^{\prime}, \beta J\right)$, on which the internal energy reads :

$$
U=-N_{\mathrm{B}}\left(2 p^{\prime}-1\right) J .
$$

Another example is provided by a Gaussian law (3.3) for $p\left(J_{k}\right)$, of variance $J$ and mean value $J_{0}$. The form (3.8) is then satisfied by a Gaussian distribution for $J_{k}$, of same variance $J$, but different mean value $J_{0}^{\prime}$. Here again, the condition $a=\beta$ defines a surface in the phase diagram, which reads :

$$
J_{0}+J_{0}^{\prime}=\beta J^{2} .
$$

One can also look for more involved examples where the $J_{k}$ 's are not independent. An example is provided by distributions previously introduced by Nishimori in reference [4]. His analysis was carried out there in Fourier variables, and is translated here in ours. This leads to generalized forms of Gaussian and binary distributions which read :

$$
P\left(J_{1}, \ldots, J_{q}\right)=C \exp -\frac{1}{2 J^{2}}\left[(q-1) \sum_{k=1}^{q} J_{k}^{2}-\sum_{k, l} J_{k} J_{l}+2 J_{0} \sum_{k} J_{k}\right] \exp \left[q\left(J_{0} / J^{2}\right) J_{q}\right]
$$


and

$$
P\left(J_{1}, \ldots, J_{q}\right)=p \delta\left(J_{q}-\frac{q-1}{q} J\right) \prod_{k=1}^{q-1} \delta\left(J_{k}+\frac{J}{q}\right)+\frac{1-p}{q-1} \sum_{l=1}^{q-1} \delta\left(J_{l}-\frac{q-1}{q} J\right) \prod_{k \neq l} \delta\left(J_{k}+\frac{J}{q}\right)
$$

respectively. The corresponding equations of the subspace $a=\beta$ are :

for (3.14) :

$$
q J_{0}=\beta J^{2}
$$

for (3.15): $\quad \frac{p(q-1)}{1-p}=\mathrm{e}^{\beta J}$.

Let us emphasize finally that the general form (3.8) for $\boldsymbol{P}$ does not allow one to consider symmetric distributions under $\mathbb{Z}_{q}$. Unfortunately, condition (3.8) also prevents a generalization of Nishimori's results to a random scalar Potts model. However we have seen that one can easily build probability distributions satisfying (3.8), depending on a few parameters only, and which could be of some physical relevance.

\section{Physical models : phase diagrams and free energies.}

4.1 General Remarks. - The exact results derived on the subspace $a=\beta$ provide interesting insights into the structure of the phase diagrams.

The first point to be discussed is the intersection of this subspace with the critical lines. It has been extensively studied by Nishimori $[1,2]$ and we simply recall the main results :

i) the absence of singularity of $U$ forbids an intersection with a first order critical variety, unless the latent heat vanishes at the intersection point.

ii) the exponent $\alpha$ of the specific heat $C$ has to be strictly negative in order to keep $C$ bounded (see Eq. (2.13)). Furthermore, if the critical boundary is differentiable, one must have $\alpha \leqslant-1$. We shall see that for most physical models, the intersection seems to be located at a tricritical point.

The location of the subspace in the phase diagram must meet the following requirements :

iii) from result (2.14) the subspace cannot lie in the spin glass phase where $m=0$ and $Q \neq 0$;

iv) the topological constraint iv) of subsection 2.2 has to be satisfied, which severely restricts the possible configurations of the phase diagram. A detailed discussion of this point can be found in [2]. The main consequence is that the boundary of the ferromagnetic phase has to be reentrant (or strictly vertical) in the variables $\left(T, p_{1}, \ldots, p_{n}\right)$ when the temperature is lower than that of the intersection point (see for instance Fig. 1a).

In the following we compare these constraints with results previously derived by either analytical or numerical methods for the phase diagrams of some physical models. We also study how the rigorous bounds for the quenched free energy obtained in subsection 2.3 apply to these specific models.

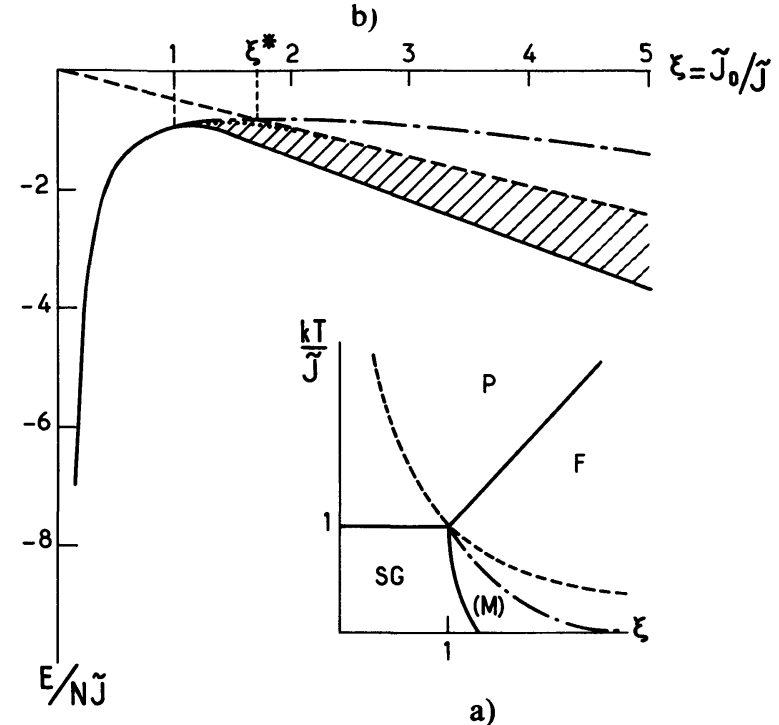

Fig. 1. - S.K. model. (a) : Phase diagram. Dashed line : Nishimori's line. Dashed dotted line : De Almeida-Thouless line. (b) : Free energy. Plain line : annealed approximation $F_{A}$. Dashed dotted line : upper bound $F_{\mathrm{S}}$. Dashed line : internal energy $U$. Dotted line : S.K. solution.

4.2 INFINITE RANGE MODELS. - These models are of particular interest since their exact solution is given by the mean field theory.

4.2.1 S.K. model. - In the Sherrington-Kirkpatrick (S.K.) model [5], Nishimori's line is defined by :

$$
\beta \tilde{\mathcal{J}}^{2}=\tilde{J}_{0}
$$

where $\tilde{J}$ and $\tilde{J}_{0}$ are the scaled parameters of the Gaussian distribution over the bonds :

$$
\begin{aligned}
\tilde{J} & =J \sqrt{N} \\
\tilde{J}_{0} & =N J_{0} .
\end{aligned}
$$

As shown by Nishimori $[1,2]$ this line intersects the boundary between paramagnetic and ferromagnetic phases precisely at the tricritical point $\left(\beta \tilde{J}=J_{0} / J=1\right)$. Moreover, the topological constraint is satisfied, since the ferro/spin glass (F/SG) boundary is reentrant (see Fig. 1a). Nishimori has also shown that there is a cancellation of singularities at the tricritical point leading to a bounded specific heat.

We now turn to the bounds on the free energies on the line. It is straightforward to obtain the expressions of the annealed free energy $F_{\mathrm{A}}$. This provides a lower bound on $F$. According to subsection 2.3, an upper bound is given by $\min \left(U, F_{\mathrm{s}}\right)$. Analytical expressions of these quantities are given in Appendix B and plotted in figure $1 \mathrm{~b}$, together with the S.K. free energy $F_{\text {S.K. }}$. Let us also note that Nishimori's line 
lies in the domain of local stability of the solution with no replica symmetry breaking (Almeida-Thouless domain [6]).

The first striking observation is that these upper and lower bounds exactly coincide in all the paramagnetic phase $\left(\xi=\tilde{J}_{0} / \tilde{J}<1\right)$. This provides a rigorous proof that the S.K. solution is the exact solution on the line in the paramagnetic phase. This proof is independent of any assumption concerning the replica symmetry breaking. It is not surprising that at high temperature $(\xi \rightarrow 0)$ the quenched solution coincides with the annealed one [7] but it is remarkable that this remains true on the line down to the tricritical point.

For $\xi>1$ (ferromagnetic phase) the two bounds split off. For $\xi^{*}=2 \sqrt{\log 2}, F_{\mathrm{s}}$ becomes greater than the internal energy which is then the relevant upper bound for $\xi>\xi^{*}$. The allowed domain for the free energy is hatched on figure 1b. At low temperature $(\xi \rightarrow \infty)$ we expect the entropy to vanish, hence the free energy to be asymptotic to $U$. This is observed for $F_{\text {S.K. }}$. which interpolates very " quickly " between $F_{\mathrm{S}}$ and $U$. Hence a good approximation to $F_{\text {S.K. }}$ is

$$
\begin{aligned}
& F=F_{\mathrm{S}} \text { for } \xi \leqslant \xi^{*} \\
& F=U(S=0) \text { for } \xi \geqslant \xi^{*} .
\end{aligned}
$$

This approximation is reminiscent of the random energy model [8].

4.2.2 Dilute models. - Recently L. Viana and A. J. Bray [9] have studied a dilute infinite range model characterized by a probability distribution over the bonds $P\left(J_{i j}\right)=\frac{p}{N} P_{p}\left(J_{i j}\right)+\left(1-\frac{p}{N}\right) \delta\left(J_{i j}\right)$. In this infinite range model the probability $p / N$ of a nonzero bond goes to zero when $N \rightarrow \infty$, while in the S.K. model it is the strength of the bonds which goes to zero in this limit. Choosing for example : $P_{p}\left(J_{i j}\right)=$ $q \delta\left(J_{i j}-J\right)+(1-q) \delta\left(J_{i j}+J\right)$ they obtained a phase diagram with three (or four) phases which can coexist along a multicritical line defined by :

$$
\tanh \left(\frac{J}{T}\right)(2 q-1)=\tanh ^{2} \frac{J}{T}=1 / p .
$$

It is remarkable to note that for this model, the subspace $a=\beta$ reads : $2 q-1=\tanh J / T$ defining a surface in the phase diagram which includes the multicritical line. By inspection of their figure 5, one can see that the topological constraint of subsection 3.1 is satisfied.

4.2.3 Potts model. - Another interesting infinite range model is the generalization of the S.K. model to Potts variables studied by Nishimori and Stephen [4]. For low values of $q$ the tricritical point is on the line, but for $q \rightarrow \infty$ this behaviour is forbidden, all transitions being of first order at this point. However the line intersects the $F / P$ boundary at a point of infinite slope, where the transition could become of second order and where the topological constraint is satisfied.

\subsection{Models WITH NEAREST NEIGHBOURS INTERAC- TIONS.}

4.3.1 3d Ising spin glass. - It is now currently admitted that a spin glass transition occurs in the $3 \mathrm{~d}$ random Ising model [10-12]. Recent numerical results obtained by different techniques of real-space renormalization are available, but only a few of them give the complete phase diagram.

For the binary distribution, we refer to the work of R. Maynard and R. Rammal [13] who suggest the values $k T_{\mathrm{c}} / J \simeq 1.8, p_{\mathrm{c}} \simeq 0.25$ for the tricritical point, which is very close to the line $2 p-1=\tanh J / k T$. Moreover their SG/F boundary is slightly reentrant, in accordance with the topological constraint.

We are led to a similar conclusion in the case of the Gaussian distribution for which the approximation procedure of B. W. Southern and A. P. Young [14] yields the values $J_{0} / J \simeq 1.61, k T_{\mathrm{c}} / J \simeq 0.70$ for the tricritical point, in good agreement with a location on the line. Here again their nearly vertical SG/F boundary satisfies the topological constraint. It would clearly be of great interest to confirm numerically the location of the tricritical point on Nishimori's line with a good accuracy.

For the sake of completeness, we give here the upperbound for $F$ on the line in the case of a binary distribution :

with :

$$
F \leqslant \min \left(U, F_{\mathrm{s}}\right)
$$

$$
\begin{aligned}
& \frac{U}{N J}=-3(2 p-1) \\
& \frac{F_{\mathrm{S}}}{N J}=\frac{4 \ln 2+3 \ln p+3 \ln (1-p)}{\ln p-\ln (1-p)} .
\end{aligned}
$$

4.3.2 2d Ising spin glass. - The existence of a spin glass transition at $T \neq 0$ in the $2 \mathrm{~d}$ random Ising model has been widely discussed. Following numerical [15] and theoretical [16] works, it is generally admitted that such a transition does not occur. Nevertheless recent simulations performed on special purpose computers show that the lower critical dimension $d_{\mathrm{c}}=2$ is not excluded [17]. Sophisticated algorithms [13] even support the existence of a new phase sharing common features with a SG phase.

It is therefore of great interest to study how the information available on Nishimori's line can shed light on this question.

i) All numerical simulations suggest an intersection point (T) of the line with the $F / P$ phase boundary for $p \simeq 0.9$. As previously discussed, this implies a second order $F / P$ transition at point $\mathrm{T}$, with $\alpha<0$. On the other hand, it has been shown by V. S. and V. I. Dotsenko [18] that for $p$ close to 1, the singularity of the specific heat on $F / P$ boundary behaves like 
$A(p) \ln \ln \left|\frac{T-T_{\mathrm{c}}}{T_{\mathrm{c}}}\right|$. Using universality arguments, these authors suggest that this result should hold for any $p$. This would be in contradiction with $\alpha<0$ unless $A(p)$ vanishes at point T. Though it is not the unique way out of this problem, a possible answer is to assume that $T$ is in fact a tricritical point where a cancellation of singularities may occur. This is the generic behaviour in the case of infinite range models.

ii) In most numerical works, the $\boldsymbol{F} / \boldsymbol{P}$ boundary does not satisfy the topological constraint iv) of subsection 2.2. At best, they give a nearly vertical boundary, but with an incorrect sign for the slope.

In the following, we suggest that it could be possible to satisfy i) and ii) simultaneously by assuming the existence of a third phase, already introduced in the numerical studies of R. Maynard, R. Rammal and J. C. Angles d'Auriac [13, 19] (random antiphase). However, though their work is a numerical evidence for a new phase, their determination of the possible tricritical point $T$ is not precise due to the large slope of the $F / P$ boundary in the vicinity of $p \simeq 0.9$. This is why we suggest that the shape of the boundaries of reference [13] be slightly modified in order to satisfy i) and ii). This leads to the phase diagram proposed in figure $2 \mathrm{a}$. The possible tricritical point $\mathrm{T}$ lies on Nishimori's line and is defined by :

$$
\frac{k T_{\mathrm{c}}}{J} \simeq 0.9 \quad p_{\mathrm{c}} \simeq 0.9
$$

This new phase could also be a new type of short range magnetic order (see for example Ref. [22]).

We now apply the bounds on the free energy $F$, given in subsection 2.3 , to the $2 \mathrm{~d}$ binary case. An explicit expression for the annealed free energy $F_{\mathrm{A}}$ can be obtained using the Onsager solution of the pure model. This lower bound and the upper ones, $U$ and $F_{\mathrm{S}}$, are given in Appendix B and plotted in figure $2 b$. The resulting constraint on $F$ is very stringent, as can be seen on the hatched region of figure $2 b$. At high temperature (paramagnetic phase) the upper and lower bounds are asymptotically equal. In fact, they coincide with a very good approximation down to $k T / J \simeq 1.6(p \simeq 0.8)$. This shows that the annealed solution is nearly exact in a wide range of temperatures in the paramagnetic phase. However, $F$ cannot be equal to $F_{\mathrm{A}}$ in all the paramagnetic phase since $F_{\mathrm{A}}$ exhibits an Onsager type transition at $p^{*} \simeq 0.82$. While simulations yield the critical value $p_{\mathrm{c}} \simeq 0.9$. This shows that the identification of $F$ with $F_{\mathrm{A}}$, which was exact in the mean field S.K. model, is only asymptotic here.

\section{Conclusion.}

We emphasize that exact properties derived on a subspace of the phase diagram provide useful infor- b)

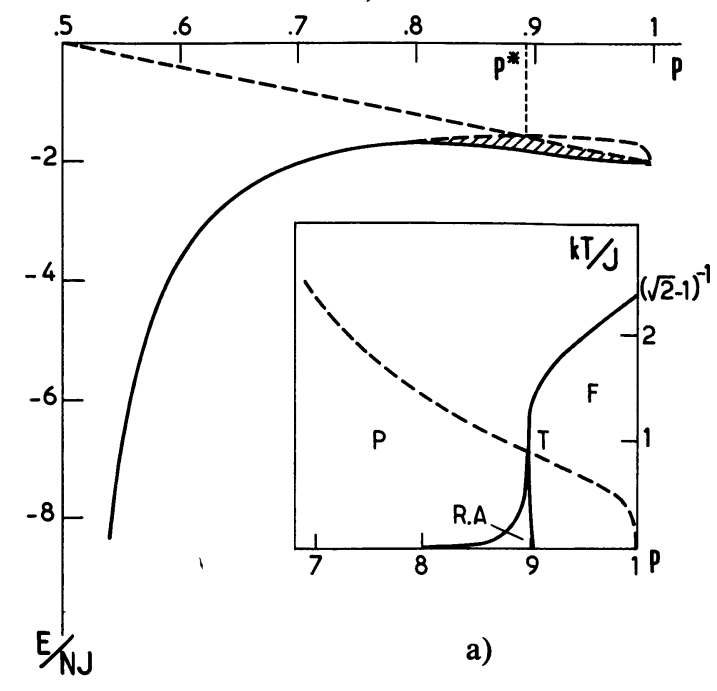

Fig. 2. - 2d binary Ising spin glass. (a) : Possible structure of phase diagram. (b) : Free energy. Plain line : annealed approximation $F_{\mathrm{A}}$. Dashed curve : upper bound $F_{\mathrm{s}}$. Dashed straight line : internal energy $U$.

mation on the general phase structure. This calls for further theoretical work related to Nishimori's line, and for a systematic comparison between numerical simulations and these exact results. In particular, the knowledge of the exact internal energy and of an upper bound for the free energy on the line, is a useful test of the accuracy of such simulations.

In view of our results, we suggest the idea that the question of the lower critical dimension for the spin glass problem could be approached by precise numerical studies in $2 \mathrm{~d}$ with special care for a possible tricritical point on this line.

In $3 \mathrm{~d}$ also, where the simulations seem to agree with the existence of a tricritical point on the line, such a method could allow its precise determination.

\section{Acknowledgments.}

We thank J. M. Maillard for his constant encouragements and his helpful suggestions. We are also grateful to G. Toulouse and R. Rammal for valuable discussions and comments. We are also indebted to E. Brezin and M. Gabay.

\section{Appendix A.}

In this appendix, we prove the results of section 2 for an arbitrary gauge group. The proof is given in detail for the points i), ii), iii) and the upper bound of subsection 2.3 , but is only sketched for iv). 
i) Simplification of the quenched energy.

The energy reads, from (2.4) :

$$
U=-\int \prod_{(i j)} \phi\left[J_{i j}\right] D J_{i j} \exp \left(a \sum_{(k l)} J_{k l}(e)\right) \frac{\sum_{\{g\}} \sum_{(k l)} J_{k l}\left(g_{k} g_{l}^{-1}\right) \mathrm{e}^{-\beta H}}{\sum_{\{g\}} \mathrm{e}^{-\beta H}} .
$$

Given a set $\left\{\xi_{i}\right\}$ of group elements, we make in the integral the change of variables $J_{i j} \rightarrow^{\xi_{i}^{-1} \xi_{j}} J_{i j}$. According to the gauge invariance of $\phi$, we get :

$$
U=-\int \prod_{(i j)} \phi\left[J_{i j}\right] D J_{i j} \exp \left[a \sum_{(k l)} J_{k l}\left(g_{k} g_{l}^{-1}\right)\right] \frac{\sum_{\{g\}} \sum_{(k l)} J_{k l}\left(\xi_{k} g_{k} g_{l}^{-1} \xi_{l}^{-1}\right) \exp \left[\beta \sum_{i j} J_{i j}\left(\xi_{i} g_{i} g_{j}^{-1} \xi_{j}^{-1}\right)\right]}{\sum_{\{g\}} \exp \left[\beta \sum_{(i j)} J_{i j}\left(\xi_{i} g_{i} g_{j}^{-1} \xi_{j}^{-1}\right)\right]} .
$$

We now make the reindexing $g_{i} \rightarrow \xi_{i} g_{i}$ in all sums over $\{g\}$ and sum over all configurations $\left\{\xi_{i}\right\}$. We obtain :

$$
U=-\frac{1}{V_{\mathrm{G}}^{N}} \int \prod_{(i j)} \phi\left[J_{i j}\right] D J_{i j} \sum_{\{\xi\}} \exp \left[a \sum_{(k l)} J_{k l}\left(\xi_{k} \xi_{l}^{-1}\right)\right] \frac{\sum_{\{g\}} \sum_{(k l)} J_{k l}\left(g_{k} g_{l}^{-1}\right) \exp \left[\beta \sum_{(i j)} J_{i j}\left(g_{i} g_{j}^{-1}\right)\right]}{\sum_{\{g\}} \exp \left[\beta \sum_{(i j)} J_{i j}\left(g_{i} g_{j}^{-1}\right)\right]}
$$

where $V_{\mathrm{G}}$ denotes the volume of the group $\mathrm{G}$. When the condition $a=\beta$ is met, the sum over $\{\xi\}$ of the numerator simplifies with the partition function of the denominator, and we get :

$$
U=-\frac{1}{V_{\mathrm{G}}^{N}} \sum_{\{g\}} \int_{(i j)}\left\{D J_{i j} \phi\left[J_{i j}\right] \mathrm{e}^{\beta J_{i j}\left(g_{i} g_{j}^{-1}\right)}\right\} \sum_{(k l)} J_{k l}\left(g_{k} g_{l}^{-1}\right) .
$$

The change of variables $J_{i j} \rightarrow{ }^{g_{i j} g^{-1}} J_{i j}$ is then used to reconstitute the original measure (2.10). This leads immediately to (2.12). The same result holds for all observables $O$ depending on the configuration of sites and bonds in a gauge invariant way, that is of the form $O\left[\left\{J_{i j}\left(g_{i} g_{j}^{-1}\right)\right\}\right]$.

ii) Bound on the specific heat.

The last remark of i) applies to the squared Hamiltonian $H^{2}$; we obtain :

$$
\left\langle H^{2}\right\rangle=N_{\mathrm{B}}\left\langle J^{2}(e)\right\rangle+N_{\mathrm{B}}\left(N_{\mathrm{B}}-1\right)\langle J(e)\rangle^{2} .
$$

The specific heat is given by :

$$
\langle C\rangle=T^{2}\left\{\left\langle H^{2}\right\rangle-\left\langle\langle H\rangle_{\mathrm{th}}^{2}\right\rangle\right\}
$$

where \langle\rangle$_{\text {th }}$ denotes the thermodynamical average over the configurations. Using the inequality $\left\langle\langle H\rangle_{\text {th }}^{2}\right\rangle \geqslant$ $\left\langle\langle H\rangle_{\text {th }}\right\rangle^{2}$ together with i), we obtain the bound (2.13) on $C$.

iii) Order parameters.

The usual definition of a ferromagnetic $(m)$ and spin glass $(Q)$ order parameters can conveniently be generalized to our model (2.1) by introducing a function $\rho$ from $\mathrm{G}$ to $\mathbb{C}$, such that :

$$
\begin{aligned}
\rho\left(g g^{\prime}\right) & =\rho(g) \rho\left(g^{\prime}\right) \\
\rho(e) & =1 \\
\frac{1}{V_{\mathrm{G}} \int D g \rho(g)} & =0
\end{aligned}
$$

$m_{i}$ and $Q_{i}$ are now defined as :

$$
m_{i}=\left\langle\left\langle\rho\left(g_{i}\right)\right\rangle_{\mathrm{th}}\right\rangle \quad Q_{i}=\left\langle\left\langle\rho\left(\dot{g}_{i}\right)\right\rangle_{\mathrm{th}}\left\langle\rho\left(g_{i}\right)\right\rangle_{\mathrm{th}}\right\rangle .
$$

As an example, for the Potts spin glass of subsection 3.2, one can choose $\rho(\theta)=\mathrm{e}^{i \theta}$. Using the same techniques 
as for i), one has :

$$
\begin{aligned}
m_{i} & =\int \prod_{(i j)} P\left(J_{i j}\right) D J_{i j} \sum_{\{g\}} \rho\left(g_{i}\right) \frac{\mathrm{e}^{-\beta H[\{g\}]}}{Z} \\
& =\frac{1}{V_{\mathrm{G}}^{N}} \int \prod_{i j} \phi\left(J_{i j}\right) D J_{i j} \sum_{\{\xi\}} \mathrm{e}^{-a H[\{\xi\}]} \sum_{\{g\}} \rho\left(g_{i} \xi_{i}\right) \frac{\mathrm{e}^{-\beta H[\{g \xi\}]}}{Z}
\end{aligned}
$$

when $a=\beta$ the usual simplification occurs :

$$
\begin{aligned}
m_{i} & =\frac{1}{V_{\mathrm{G}}^{N}} \int \prod_{i j} \phi\left(J_{i j}\right) D J_{i j} \sum_{\{\xi\}} \rho\left(\xi_{i}\right) \mathrm{e}^{-\beta H[\{\xi]} \sum_{\{g\}} \rho\left(g_{i}\right) \frac{\mathrm{e}^{-\beta H[\{g\}]}}{Z} \\
& =\frac{1}{V_{\mathrm{G}}^{N}} \int \prod_{i j} \phi\left(J_{i j}\right) D J_{i j} \sum_{\left\{g^{\prime}\right\}} \mathrm{e}^{-\beta H\left[\left\{g^{\prime}\right\}\right]} \sum_{\{\xi\}} \rho\left(\xi_{i}\right) \frac{\mathrm{e}^{-\beta H[\{\xi]}}{Z} \sum_{\{g\}} \rho\left(g_{i}\right) \frac{\mathrm{e}^{-\beta H[\{g\}]}}{Z} .
\end{aligned}
$$

Reindexing the sum over $\left\{g^{\prime}\right\}$, one obtains $m_{i}=Q_{i}$ on the subspace. In the same way, it can be shown that the whole hierarchy of parameters $Q_{i}^{(n)}=\left\langle\left\langle\rho\left(g_{i}\right)\right\rangle_{\mathrm{th}}^{n}\right\rangle$ verifies $Q_{i}^{(2 n)}=Q_{i}^{(2 n-1)}$.

iv) Topological constraint.

A full proof of this point in the case of $\mathbb{Z}_{2}$ has been given by Nishimori [2]. It is based on a generalization of an inequality due to Horiguchi and Morita [20]. This inequality can easily be generalized to our model, and reads :

$$
\left|m_{i}\left(\beta ; p_{1}, \ldots, p_{n}\right)\right| \leqslant K\left\langle\left|\left\langle\rho\left(g_{i}\right)\right\rangle_{\mathrm{th}}\right|\right\rangle\left(\beta^{*} ; p_{1}, \ldots, p_{n}\right)
$$

where $K$ is a constant, and $\beta^{*}=a\left(p_{1}, \ldots, p_{n}\right)$, as was defined in the text. When the point $\left(\beta^{*} ; p_{1}, \ldots, p_{n}\right)$ of the subspace is in the paramagnetic phase, the R.H.S. is zero, and therefore $m\left(\beta, p_{1}, \ldots, p_{n}\right)$ vanishes for all temperatures.

v) Bound on the free energy.

We derive expression $(2.15)$ for $\left\langle Z^{-1}\right\rangle$ :

$$
\left\langle Z^{-1}\right\rangle=\int \prod_{i j} \phi\left(J_{i j}\right) D J_{i j} \exp \left[a \sum_{i j} J_{i j}(e)\right]\left[\sum_{\{g\}} \exp \left[\beta \sum_{i j} J_{i j}\left(g_{i} g_{j}^{-1}\right)\right]\right]^{-1} .
$$

As for i), we make the change of variables $J_{i j} \rightarrow \xi_{i}^{-1} \xi_{j} J_{i j}$, reindex the sum over $\{g\}$, and sum over all $\left\{\xi_{i}\right\}$. This leads to :

$$
\left\langle Z^{-1}\right\rangle=\frac{1}{V_{\mathrm{G}}^{N}} \int \prod_{i j} \phi\left(J_{i j}\right) D J_{i j} \sum_{\{\xi\}} \exp \left[a \sum_{i j} J_{i j}\left(\xi_{i} \xi_{j}^{-1}\right)\right]\left[\sum_{g} \exp \left[\beta \sum_{i j} J_{i j}\left(g_{i} g_{j}^{-1}\right)\right]\right]^{-1} .
$$

On the subspace $a=\beta$, this reduces to formula (2.15).

\section{Appendix B.}

Here we give the expressions of the annealed free energy $F_{\mathrm{A}}$ and of the upper bound min $\left(U, F_{\mathrm{S}}\right)$ on $\cdot$ Nishimori's line, for S.K. and $2 \mathrm{~d}$ binary Ising models.

1. S.K. MODEL. - Using the steepest descent method [5], one readily obtains, with $\xi=\tilde{J}_{0} / \tilde{J}$ :

$$
\begin{aligned}
& \frac{F_{\mathrm{A}}}{N \tilde{J}}=-\frac{\ln 2}{\xi}-\frac{\xi}{4} \quad \text { for } \quad \xi \leqslant 1 \\
& \frac{F_{\mathrm{A}}}{N \tilde{J}}=-\frac{\ln 2}{\xi}-\frac{\xi}{4}+\frac{x(\xi)}{2 \xi}-\frac{1}{\xi} \ln [\cosh \xi x(\xi)] \quad \text { for } \quad \xi \geqslant 1
\end{aligned}
$$

where $x(\xi)$ is the non-zero solution of :

$$
x=\xi \tanh (\xi x)
$$


$U$ is simply given by :

$$
\frac{U}{N \widetilde{J}}=-\frac{\xi}{2}
$$

and $\frac{F_{\mathrm{S}}}{N \widetilde{J}}=-\frac{\ln 2}{\xi}-\frac{\xi}{4}$ which coincides with $F_{\mathrm{A}}$ for $\xi \leqslant 1$.

Using the equality $Q=m$ on the line, one easily obtains the expression of the S.K. solution :

$$
\frac{F_{\text {S.K. }}}{N \tilde{J}}=-\frac{\xi}{4}(1-Q)^{2}+\xi \frac{Q^{2}}{2}-\frac{1}{\sqrt{2 \pi}} \int_{-\infty}^{+\infty} \frac{\mathrm{d} z \mathrm{e}^{-z^{2} / 2}}{\xi} \ln 2 \cosh \left(z \xi \sqrt{Q}+\xi^{2} Q\right)
$$

where $Q(\xi)$ is the solution of

$$
Q(\xi)=\frac{1}{\sqrt{2 \pi}} \int_{-\infty}^{+\infty} \mathrm{d} z \mathrm{e}^{-z^{2} / 2} \tanh \left[z \xi \sqrt{Q}+\xi^{2} Q\right] .
$$

2. 2d BINARY IsING SPIN GLASS. - The annealed solution can easily be obtained from the Onsager solution of the pure model. On the line, this leads to :

$$
\begin{aligned}
& \frac{F_{\mathrm{A}}}{N J}=\frac{1}{\ln \frac{p}{1-p}}\left\{-2 \ln 2+2 \ln \left(1-(2 p-1)^{2}\right)-\right. \\
& \left.\quad-\frac{1}{(2 \pi)^{2}} \int_{0}^{2 \pi} \int_{0}^{2 \pi} \mathrm{d} q_{1} \mathrm{~d} q_{2} \ln \left[\left(1+(2 p-1)^{4}\right)^{2}-2(2 p-1)^{2}\left(1-(2 p-1)^{4}\right)\left(\cos q_{1}+\cos q_{2}\right)\right]\right\}
\end{aligned}
$$

$U$ is given by :

$$
U / N J=-2(2 p-1)
$$

and

$$
\frac{F_{\mathrm{S}}}{N J}=\frac{2}{\ln \frac{p}{1-p}} \ln 2 p(1-p)
$$

\section{References}

[1] Nishimori, H., J. Phys. C 13 (1980) 4071.

[2] Nishimori, H., Prog. Theor. Phys. 66 (1981) 1169.

[3] Georges, A., Hansel, D., Le Doussal, P., J. Physique 46 (1985) 1309.

[4] Nishimori, H., Stephen, M. J., Phys. Rev. B 27 no 9 (1983) 5644.

[5] Sherrington, D., KirkPatrick, S., Phys. Rev. Lett. 35 no $^{\circ} 6$ (1975) 1792.

[6] De Almeida, J., Thouless, D. J., J. Phys. A 11 (1977) 983.

[7] Derrida, B., Toulouse, G., VI Brazilian Symposium on Theoretical Physics. Erasmo Ferreira, Belita Koller, Ed. (1981).

[8] Derrida, B., Phys. Rev. B 24 no 5 (1981) 2613.

[9] Viana, L., Bray, A. J., Manchester University Preprint TH 84/13 (1984).

[10] Bray, A. J., Moore, M. A., Phys. Rev. B 31 no 1 (1985) 631.

[11] Bhatt, R. N., Young, A. P., Imperial College Preprint (1984).
[12] Ogielsky, A., Morgenstern, I., Phys. Rev. Lett. 54 $\mathrm{n}^{\circ} 9$ (1985) 928.

[13] Maynard, R., Rammal, R., J. Physique Lett. 43 (1982) L-347.

[14] Southern, B. W., Young, A. P., J. Phys. C 10 (1977) 2179.

[15] Morgenstern, I., Binder, K., Phys. Rev. Lett. 4321 (1979) 1615

[16] Hoever, P., Zittartz, J., Z. Phys. B 49 (1982) 39.

[17] Morgenstern, I., Communication to the CECAM meeting (Paris) 1985. To be published.

[18] Dotsenko, V. S., Dotsenko, V. I., J. Phys. C 15 (1982) 485 ; Sov. Phys. JETP 56 (1982) 406.

[19] Angles D'Auriac, J. C., Maynard, R., Solid State Commun. $49 \mathrm{n}^{\circ} 8$ (1984) 785.

[20] Horiguchi, Morita, J. Phys. A 15 (1982) L-75.

[21] For a short review, see JAECKEL, M. T. and MAILlaRD, J. M., J. Phys. A 18 (1985) 1229.

[22] Hälg, B., Furrer, A. and Vogt, O., Phys. Rev. Lett. 54 no 13 (1985) 1388. 